\title{
Enterocutaneous fistula: a novel video-assisted approach
}

\author{
Hugo Palma Rios ${ }^{1}$, André Goulart ${ }^{1}$, Carla Rolanda ${ }^{2,3,4}$, Pedro Leão ${ }^{1,3,4}$ \\ 'Department of General Surgery, Hospital de Braga, Braga, Portugal \\ ${ }^{2}$ Department of Gastroenterology, Hospital de Braga, Braga, Portugal \\ ${ }^{3}$ Surgical Sciences Research Domain, Life and Health Sciences Research Institute (ICVS), School of Health Sciences, University of Mino, \\ de Gualtar Campus, Braga, Portugal \\ ${ }^{4}$ ICVS/3B's - PT Government Associate Laboratory, Braga, Guimarães, Portugal
}

Videosurgery Miniinv 2017; 12 (3): 297-300

DOI: https://doi.org/10.5114/wiitm.2017.67780

\begin{abstract}
Video-assisted anal fistula treatment (VAAFT) is a novel minimally invasive and sphincter-saving technique to treat complex anal fistulas described by Meinero in 2006. An enterocutaneous fistula is an abnormal communication between the bowel and the skin. Most cases are secondary to surgical complications, and managing this condition is a true challenge for surgeons. Postoperative fistulas account for 75-85\% of all enterocutaneous fistulas. The aim of paper was to devise a minimally invasive technique to treat enterocutaneous fistulas. We used the same principles of VAAFT applied to other conditions, combining endoluminal vision of the tract with colonoscopy to identify the internal opening. We present a case of a 78-year-old woman who was subjected to a total colectomy for cecum and sigmoid synchronous adenocarcinoma. The postoperative course was complicated with an enterocutaneous fistula, treated with conservative measures, which recurred during follow-up. We performed video-assisted fistula treatment using a fistuloscope combined with a colonoscope. Once we identified the fistula tract, we performed cleansing and destruction of the tract, applied synthetic cyanoacrylate and sealed the internal opening with clips through an endoluminal approach. The patient was discharged 5 days later without complications. Two months later the wound was completely healed without evidence of recurrence. This procedure represents an alternative treatment for enterocutaneous fistula using a minimally invasive technique, especially in selected patients not able to undergo major surgery.
\end{abstract}

Key words: mini-invasive endoscopy, enterocutaneous fistula, video-assisted anal fistula treatment.

\section{Introduction}

An enterocutaneous fistula is an abnormal communication between the small or large bowel and the skin, with external drainage of intestinal contents. Postoperative fistulas account for $75-85 \%$ of all enterocutaneous fistulas. Most cases are secondary to surgical complications, with significant morbidity and mortality. Only one third of enterocutaneous fistulas close spontaneously, and the management of this condition is a true challenge for surgeons $[1,2]$.
Video-assisted anal fistula treatment (VAAFT) is a novel minimally invasive and sphincter-saving technique for treatment of complex anal fistulas described by Meinero and Mori in 2006 [3].

\section{Case report}

We present a case of a 78-year-old woman who underwent a laparoscopic total colectomy with an ileorectal lateral-to-end anastomosis using a $25 \mathrm{~mm}$ circular stapler (Ethicon, Somerville, NJ,

\section{Address for correspondence}

Pedro Leão MD, PhD, FACS, Department of General Surgery, Hospital de Braga, Campus de Gualtar, 4710-057 Braga, Portugal,

phone: +35 1915303818, e-mail: pedroleao@med.uminho.pt 
USA) for synchronous tumors of the sigmoid and caecum.

Postoperative surveillance was complicated by an anastomotic leak with consequent drainage through incision. After 2 weeks of conservative treatment the patient was discharged. Three months later, the patient presented with an abscess in the same location that was drained. In the $9^{\text {th }}$ month after surgery the patient developed an enteric drainage where the drain was placed (Photo 1). The abdominal computed tomography (CT) scan revealed an enterocutaneous fistula (Photo 2). A minimally invasive technique for fistula correction was proposed to the patient to avoid additional morbidity. After explaining the technique to the patient, informed consent was obtained.

\section{Surgical technique}

The patient was placed in the Lloyd-Davis position and the monitor of the fistuloscope was positioned opposite to the surgeon (on the contralateral side of the fistula). The monitor of the flexible endoscope for the rectal approach was positioned on the same patient's bedside as the fistuloscope's monitor, the left side for both in this case.

The technique was a combination of a transabdominal and rectal endoluminal approach, both taking place at the same time. The first step was an approach of the inner orifice with the endoscope, so a cautious evaluation of the rectal mucosa and anastomosis was made and then the endoscope was positioned around the suspicious area. The abdominal part started with the channeling of the fistula with a hydrophilic guide-wire (Terumo) under rectal en-

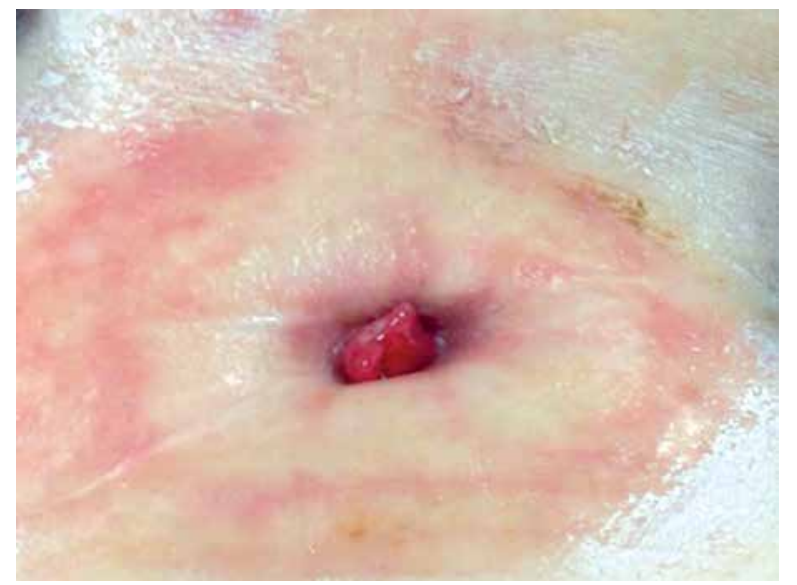

Photo 1 . Outer orifice in the right lower quadrant of the abdomen doscopic control. Then, using a Seldinger technique the fistuloscope, manufactured by Karl Storz GmbH (Tuttlingen, Germany), was inserted through the guide wire. The obturator was visible at the lower edge of the screen to ensure the correct orientation of the fistuloscope.

Similar to anal fistula, sometimes the external opening is surrounded by very tough scar tissue, and in the present case we proceeded to remove it in order to allow easier entry of the fistuloscope. Then, we simply advanced the fistuloscope along the pathway using slow movements, left/right and up/down. These maneuvers allowed the fistula to accommodate the fistuloscope while it was being straightened. Optimal vision of the lumen of the fistula was ensured by the continuous jet of irrigation solution reaching as far as the internal opening, with continuous visualization of the endoluminal side by the gastroenterologist (Photo 3).

The next goal was the destruction of the fistula from the inside with cleansing of the fistula tract and finally closure of the internal and external openings. First, we removed the obturator and replaced it with the electrode, which destroyed the fistula tract under continuous direct vision. We proceeded centimeter by centimeter from the internal to the external opening, cauterizing all fragments of the whitish materials adhering to the fistula wall and taking care not to overlook any abscess cavities or secondary fistula tract. Continuing under direct vision, necrotic materials were removed with an endo-brush. The continuous jet of irrigation solution also ensured that some waste material was eliminated into the

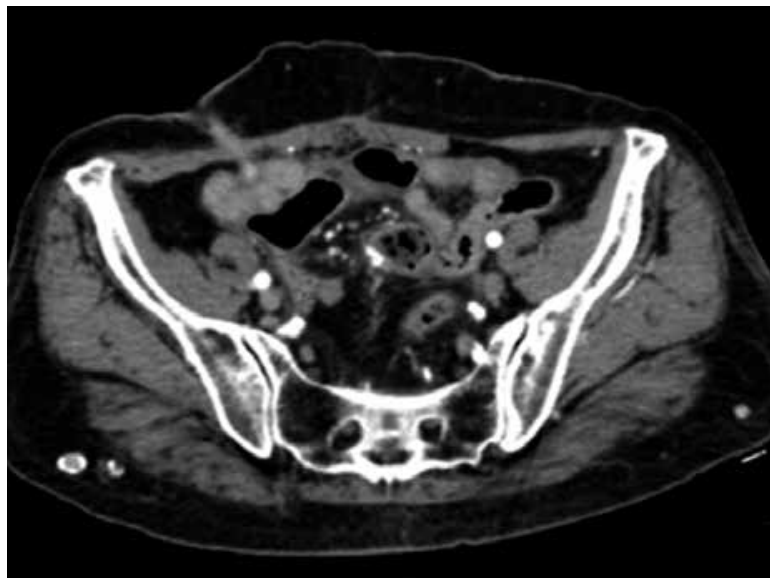

Photo 2. Computed tomography scan showing the fistula tract 

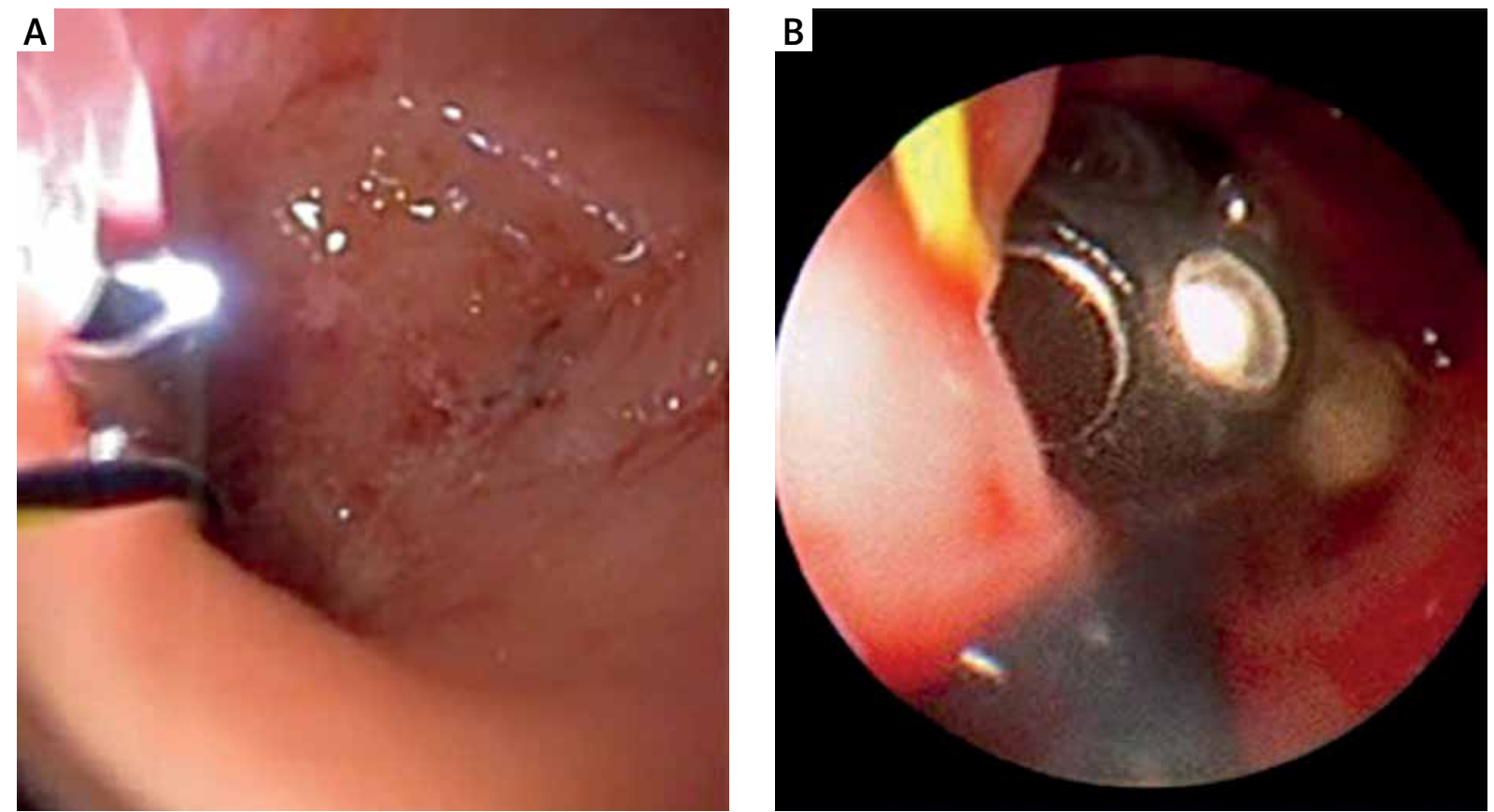

Photo 3. Double vision provided by the endoscope while the fistuloscope goes through the fistula tract

rectum through the internal opening. At this point, the gastroenterologist brushed the inner orifice, through the scope, reinforcing the ablation of the epithelial lining in this location, and applied some endoclips to close it.

After concluding this phase, we removed the fistulous tract up to the fascia and additionally applied $0.5 \mathrm{ml}$ of synthetic cyanoacrylate using a tiny catheter through the fistula (contrary to the Meinero technique). The skin was then closed with non-absorbable monofilament. The procedure and the postoperative period were uneventful, without significant pain or other complaints.

The patient underwent a 6-month follow-up without recurrence of the fistula (Photo 4).

\section{Discussion}

An enterocutaneous fistula is still one of the most complex and challenging complications in surgical practice [4-7]. In the literature an overall spontaneous closure rate of $7-70 \%$ is reported $[8,9]$. The optimal timing for a definite surgical treatment is at least 6 months after the last laparotomy or any sign of intra-abdominal infection [4]. Not all patients are able to overcome complex surgery. Minimally invasive approaches have arisen as an alternative way for these patients, with less morbidity and faster

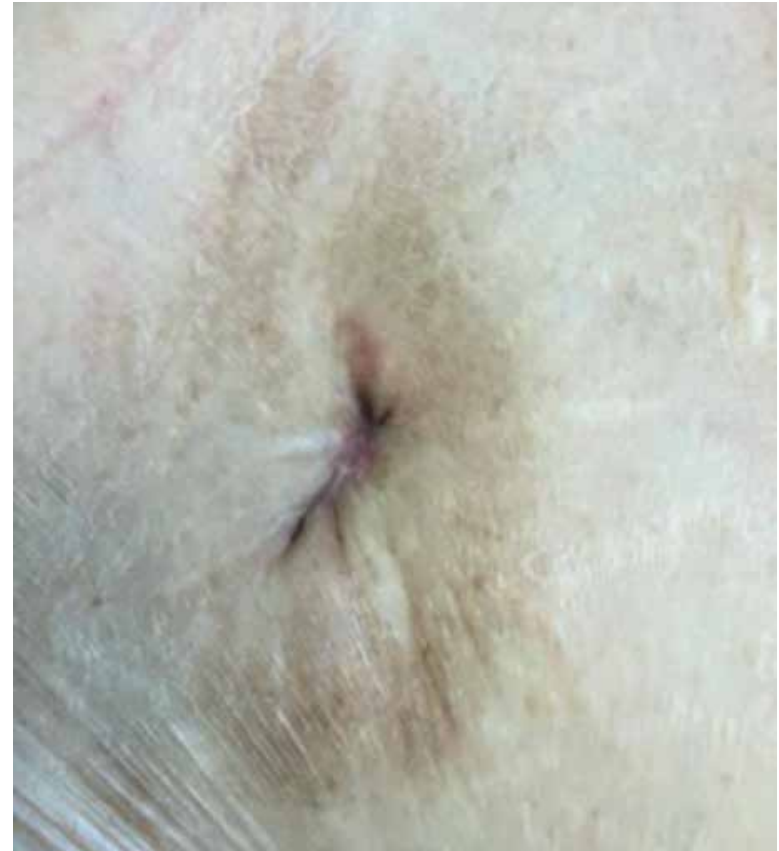

Photo 4. Surgical wound after 6 months

recovery. There are only a few published accounts on minimally invasive techniques, so it is difficult to conclude on the success rate.

One of the crucial and difficult steps of the procedure described above is to identify the fistula tract and to avoid its disruption, which can cause drain- 
age of enteric contents to the abdominal cavity. The use of a guide wire and direct vision of the inner orifice allow the correct identification of the tract.

This case was our first attempt with this combined approach. As we expected, the procedure was well tolerated and the patient had a short time of recovery. The endoscopic view is a fundamental part of the technique for guidance and to close the inner orifice. An adequate previous imaging study is another important step to achieve a good outcome because the location, shape and length of the tract are the main criteria for this approach.

\section{Conclusions}

This technique can provide an alternative for the treatment of fistula in selected patients who are not able to undergo aggressive surgery. More cases are needed to verify and improve this procedure.

\section{Conflict of interest}

The authors declare no conflict of interest.

\section{References}

1. Karvonen JA, Grönroos JM, Nikulainen V, et al. Endoscopic treatment of internal gastrointestinal fistulas with fibrin glue. Surg Laparosc Endosc Percutan Tech 2013; 23: 37-40.

2. Avalos-González J, Portilla-de Buen E, Leal-Cortés CA, et al. Reduction of the closure time of postoperative enterocutaneous fistulas with fibrin sealant. World J Gastroenterol 2010; 16: 2793-800.

3. Meinero P, Mori L. Video-assisted anal fistula treatment (VAAFT): a novel sphincter-saving procedure for treating complex anal fistulas. Tech Coloproctol 2011; 15: 417-22.

4. Bemelman WA, Boermeester, MA. Enterocutaneous fistulas. In: Complexities in Colorectal Surgery. Steele SR, Maykel JA, Champagne BJ, Orangio GR (eds.). Springer, New York 2014; 105-19.

5. Schecter WP. Management of enterocutaneous fistulas. Surg Clin North Am 2011; 91: 481-91.

6. Chapman R, Foran R, Dunphy JE. Management of intestinal fistulas. Am J Surg 1964; 108: 157-64.

7. Schein M. What's new in postoperative enterocutaneous fistulas? World J Surg 2008; 32: 336-8.

8. Lloyd DAJ, Gabe SM, Windsor ACJ. Nutrition and management of enterocutaneous fistula. Br J Surg 2006; 93: 1045-55.

9. Hollington P, Mawdsley J, Lim W, et al. An 11-year experience of enterocutaneous fistula. Br J Surg 2004; 91: 1646-51.

Received: 15.01.2017, accepted: 19.03.2017. 